\title{
Biomechanical effect of cage position combined with bilateral pedicle screw fixation in the osteoporotic lumbar spine of oblique lumbar interbody fusion: a finite element analysis
}

\section{Yichuan Qin}

Second Hospital of Shanxi Medical University

\section{Bin Zhao}

Second Hospital of Shanxi Medical University

Jie Yuan

Second Hospital of Shanxi Medical University

\section{Chaojian Xu}

Second Hospital of Shanxi Medical University

Junqiang Su

Second Hospital of Shanxi Medical University

Jiaqi Hao

Second Hospital of Shanxi Medical University

Jie LV

Second Hospital of Shanxi Medical University

Yongfeng Wang ( $\nabla$ wyfwf8@163.com )

Second Hospital of Shanxi Medical University

\section{Research Article}

Keywords: finite element analysis, OLIF, cage position, osteoporosis, bilateral pedicle screw fixation

Posted Date: March 1st, 2021

DOI: https://doi.org/10.21203/rs.3.rs-252095/v1

License: (c) (i) This work is licensed under a Creative Commons Attribution 4.0 International License. Read Full License 


\section{Abstract}

Background: The influence of cage position on postoperative cage subsidence has been paid increasing attention. The best cage position in oblique lumbar interbody fusion (OLIF) is still unclear. This study aimed to evaluate the biomechanical effects of different cage positions with stand-alone (SA) methods and bilateral pedicle screw fixation (BPSF) in the osteoporotic lumbar spine after OLIF.

Methods: A finite element (FE) model of an intact L3-L5 lumbar spine was constructed. After validation, an osteoporosis model (OP) was constructed by assigning osteoporotic material properties. SA models (SA1, SA2, SA3) and BPSF models (BPSF1, BPSF2, BPSF3) in which a cage was placed in the anterior, middle and posterior third of the L5 superior endplate (SEP) were constructed at the L4-L5 segment of the OP. The L4-L5 range of motion (ROM), the stress of the L5 SEP, the stress of the cage and the stress of fixation were compared among the different models.

Results: According to the degree of ROM of L4-L5, the stress of the L5 SEP and the stress of the cage for most physiological motions, the SA and BPSF models were ranked as follows: SA2\SA1『SA3, BPSF2】 BPSF1 $\triangle B P S F 3$. In BPSF2, the stress of fixation was minimal in most motions. At the same cage position, the ROM of L4-L5, the stress of the L5 SEP and the stress of the cage in the BPSF models were significantly reduced compared with those in SA models; compared with SA2, BPSF2 had a maximum reduction of $83.24 \%, 70.71 \%$ and $73.52 \%$ in these parameters, respectively.

Conclusions: Placing the cage in the middle third of the L5 SEP for OLIF could reduce the maximum stresses of the L5 SEP, the cage and the fixation, which may reduce the risk of postoperative cage subsidence, endplate collapse and fixation fracture in the osteoporotic lumbar spine. Compared with SA OLIF, BPSF could provide sufficient stability for the surgical segment and may reduce the incidence of the aforementioned complications.

\section{Background}

Lumbar interbody fusion (LIF) is the classic technique used to treat lumbar degenerative disease and can be achieved through multiple approaches, and each approach has advantages and disadvantages. With the development of precise and minimally invasive surgical techniques, many minimally invasive LIF procedures have emerged. In recent years, oblique lateral interbody fusion (OLIF) has been highly praised because it has been reported to yield great surgical results ${ }^{[1-2]}$. However, it is worth noting that the incidence of postoperative cage subsidence and even endplate collapse due to osteoporosis or excessive injury of the endplate is approximately $8.3 \% \sim 9.4 \%{ }^{[1.3]}$. A relevant anatomical study ${ }^{[4]}$ confirms that the width of the epiphyseal rim varies across regions. The width of the rims in the lateral region of the endplate, in the anterior region and in the posterior region are approximately $7.3 \mathrm{~mm}, 5.6 \mathrm{~mm}$ and 3.3 $\mathrm{mm}$, respectively. Therefore, the contact area between the OLIF cage and the epiphyseal ring varies by the cage position, but few studies on the effect of cage position on the biomechanics of the lumbar spine have been conducted in osteoporosis patients. 
In this study, OLIF osteoporosis models with different cage positions, stand-alone (SA) methods and bilateral pedicle screw fixation (BPSF) were created. By analyzing the lumbar biomechanics, we can theoretically select a more reasonable cage position, identify the advantages of BPSF in the osteoporotic lumbar spine and provide reference information for clinicians.

\section{Materials And Methods}

\subsection{Construction of the normal intact lumbar finite element model and osteoporosis model}

In this study, we selected an adult male with no history of lumbar disease. CT scans of his lumbar spine were performed with 0.625 -mm-thick slices (Siemens, Germany). The data obtained in the DICOM format were imported into Mimics 17.0 (Materialise, Leuven, Belgium) to reconstruct the L3-L5 model. Next, the L3-L5 model was imported into Geomagic Wrap 2017 (3D Systems, Inc., South Carolina, USA) for hole filling, smoothing, polishing, simplification, surface construction and other processing steps. Then, cancellous bone, cortical bone, intervertebral discs, cartilage endplates and facet joints were reconstructed in SolidWorks 2016 (SolidWorks Corporation, Concord, MA, USA). Finally, the normal intact lumbar finite element (FE) model was constructed by adding ligaments, separating meshes and assigning material properties in ANSYS Workbench 16.0(ANSYS, Ltd., Canonsburg, Pennsylvania, USA)Figure 1区.

The nucleus pulposus accounted for $30 \%-50 \%$ of the disc volume ${ }^{[6]}$. The thickness of the cartilage endplate and cortical bone was $1 \mathrm{~mm}$, and the thickness of the facet joints was $0.2 \mathrm{~mm}^{[7]}$. Spinal ligaments (including the anterior longitudinal ligament, posterior longitudinal ligament, flava ligament, interspinous ligament, supra-spinous ligament, intertransverse ligament and facet capsular ligament) were modeled by spring units that were subjected to only tension. The material properties of the models were derived from previous studies ${ }^{[8-10]}$ (Table 1$)$. 
Table 1

Material properties used in the models

\begin{tabular}{|lll|}
\hline Component/Materials & Young Modulus E (MPa) & Poisson's Ratio \\
\hline Cortical bone & $\begin{array}{l}12000 \text { (normal)/ } \\
8040 \text { (osteoporosis) }\end{array}$ & 0.3 \\
\hline Cancellous bone & $\begin{array}{l}100 \text { (normal)/ } \\
34 \text { (osteoporosis) }\end{array}$ & 0.2 \\
\hline Posterior elements & 3500 (normal)/ & 0.3 \\
\hline Cartilage endplate & 2345 (osteoporosis) & \\
\hline Articular cartilage & 2000 & 0.2 \\
\hline Annulus fibrosus & 25 & 0.25 \\
\hline Nucleus pulposus & 4.2 & 0.45 \\
\hline Ligaments & 1 & 0.499 \\
\hline Anterior longitudinal & 7.8 & 0.3 \\
\hline Posterior longitudinal & 10 & 0.3 \\
\hline Intertransverse & 10 & 0.3 \\
\hline Ligamentum flavum & 15 & 0.3 \\
\hline Interspinous & 8 & 0.3 \\
\hline Supraspinous & 8 & 0.3 \\
\hline Capsular & 7.5 & 0.3 \\
\hline Cage (PEEK) & 3600 & \\
\hline Screws and rods (titanium) & 110000 & \\
\hline & & 0.3 \\
\hline
\end{tabular}

The osteoporosis model (OP) were modified from the normal intact model by adjusting material properties. Osteoporosis was induced by reducing the elastic moduli of the cancellous bone by $66 \%$ and those of the cortical shell, endplates, and posterior elements by $33 \%{ }^{[5]}$. The soft tissue structures remained unchanged (Table 1).

\subsection{Construction of the OLIF FE model}

The cage and BPSF were created in SolidWorks 2016 (Fig. 2). The length, width and height of the cage were $50 \mathrm{~mm}, 17 \mathrm{~mm}$ and $10 \mathrm{~mm}$, respectively, with an 8-degree sagittal angle. The rectangular plane 
instead of the serrated plane was used. The length of the pedicle screws was $50 \mathrm{~mm}$, and the diameter of these screws was $6.5 \mathrm{~mm}$. The rods were $53 \mathrm{~mm}$ in length and $5.5 \mathrm{~mm}$ in diameter. The OP was assembled with the cage and BPSF. Moro et al. ${ }^{[11]}$ divided the area between the anterior and posterior edges of the vertebral body into zones I, II, III, and IV. In this study, the L5 superior endplate (SEP) was equally divided into four parts, and the demarcation points were recorded as $a, b$ and $c$. The intersection of the maximum transverse and longitudinal diameter of the cage was recorded as central point $A$. The long axis of the cage was placed parallel to the transverse diameter of the L5 SEP, and A overlapped with $a, b$ and $c$ (Fig. 3). Then, SA models (SA1, SA2, SA3) and BPSF models (BPSF1, BPSF2, BPSF3) with a

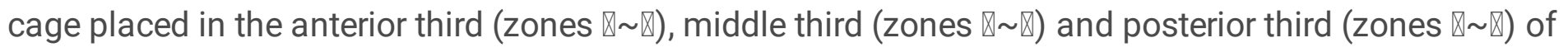
the L5 SEP were created (Fig. 4).

In all OLIF models, L4-L5 was considered the operative segment, and the cage was implanted as a single unit on the left side. The nucleus pulposus, annulus fibrous and cartilage endplate were removed. The cage and BPSF material properties are listed in Table 1.

\subsection{Boundary conditions, loading methods and biomechanical evaluation}

The contact type of facet joints was set to "frictional", and the frictional coefficient was 0.2 ; the contact types of the other components were set to "bonded". The inferior surface of L5 was fixed. An axial preload of $400 \mathrm{~N}$ and a $10 \mathrm{Nm}$ moment were imposed on the superior surface of $\mathrm{L} 3$ to simulate 6 physiological motions: flexion/extension, right/left lateral bending and right/left axial rotation ${ }^{[12-13]}$. The range of motion (ROM) of L4-L5, the stress of the L5 SEP, the stress of the cage and the stress of the fixation were analyzed and compared to investigate the biomechanical effects of different cage positions with SA and BPSF.

\section{Results}

\subsection{Model validation}

The ROMs (L3-L4 and L4-L5) were compared with the results of previous cadaveric study ${ }^{[14]}$ and FE study ${ }^{[15]}$ (Fig. 5). We found that our results were close to theirs. Therefore, the normal intact lumbar FE model in the current study could be used for further analysis.

\subsection{L4-5 ROM}

Compared with the ROM of the OP, that of SA1 was more than $28.29 \%$ smaller, and the ROMs of SA2 and SA3 were more than $43.06 \%$ and $39.83 \%$ smaller, respectively, during all motions. The SA models listed in

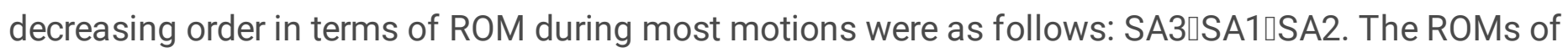
BPSF1, BPSF2 and BPSF3 were more than $80.56 \%, 80.92 \%$ and $84.13 \%$ smaller, respectively, during all motions. The BPSF models listed in decreasing order in terms of ROM during all motions except for extension were as follows: BPSF3 > BPSF1 > BPSF2 (Fig. 6).

ROM differed the most between BPSF1 and SA1 in flexion, as ROM in BPSF1 was $89.37 \%$ smaller. ROM differed the most between BPSF2 and SA2 in flexion, as ROM in BPSF2 was 83.24\% smaller. ROM 
differed the most between BPSF3 and SA3 in flexion, as ROM in BPSF3 was $74.28 \%$ smaller.

\subsection{Stress of the L5 SEP}

The SA models listed in decreasing order in terms of the maximum Von Mises stress of the L5 SEP during all motions except extension were as follows: SA3 > SA1 > SA2. The stresses of SA1 and SA2 were the highest in flexion, and the stress of SA3 in flexion was second only to that in right lateral bending, which reached 79.39 $\mathrm{MPa}$. The BPSF models listed in decreasing order in terms of the maximum Von Mises stress of the L5 SEP were as follows: BPSF3 > BPSF1 > BPSF2. The stresses of BPSF1, BPSF2 and BPSF3 in flexion and right lateral bending were significantly higher than those in other motions, while the stresses were the lowest in extension (Fig. 7).

The L5 SEP maximum stress differed the most between BPSF1 and SA1 in extension, as the stress was 71.98\% lower in BPSF1. Compared with the L5 SEP maximum stress of SA2, that of BPSF2 differed the most in extension, as it was $70.71 \%$ smaller. The L5 SEP maximum stress of BPSF3 differed the most from that of SA3 in extension, as it was $53.13 \%$ smaller. The maximum Von Mises stress of the L5 SEP was mainly concentrated in the region of the epiphyseal ring of the endplate in contact with the cage. Figure 8 shows the cloud map of L5 SEP stress in the OLIF models in flexion.

\subsection{Stress of the cage}

The SA models listed in decreasing order in terms of the maximum Von Mises stress of the cage in all motions except extension were as follows: SA3ロSA1 SA2. The stresses of SA1, SA2, and SA3 were the highest in flexion and lowest in left axial rotation. The BPSF models listed in decreasing order in terms of the maximum Von Mises stress of the cage were as follows: BPSF3 > BPSF1 > BPSF2. The stresses of BPSF1, BPSF2, and BPSF3 were the highest in flexion and lowest in extension (Fig. 9).

The cage maximum Von Mises stress of BPSF1 differed the most from that of SA1 in extension, as it was $78.38 \%$ smaller. Compared with the cage maximum Von Mises stress of SA2, that of BPSF2 differed the most in extension, as it was $73.52 \%$ smaller. The cage maximum Von Mises stress of BPSF3 differed the most from that of SA3 in extension, as it was $54.16 \%$ smaller. The maximum Von Mises stress of the cage was mainly concentrated in the region in contact with the epiphyseal ring of the endplate. Figure 10 shows the cloud map of cage stress in the OLIF models in flexion.

\subsection{Stress of the fixation}

The BPSF models listed in decreasing order in terms of the maximum Von Mises stress of the fixation were as follows: BPSF3 > BPSF2 > BPSF1 in flexion and BPSF1 > BPSF2 > BPSF3 in extension. The fixation stress of BPSF2 was the lowest in other motions (Fig. 11). The maximum Von Mises stress of fixation was mainly concentrated at the junction of the pedicle screws and rods. Figure 12 shows the cloud map of fixation stress in the BPSF models in left axial rotation.

\section{Discussion}


Lumbar interbody fusion (LIF) is an effective treatment for various spinal disease ${ }^{[16-17]}$ and is the most widely used surgical procedure ${ }^{[18]}$, but complications such as excessive blood loss, muscle denervation, iatrogenic muscle disorders and soft tissue injury are unavoidable ${ }^{[2]}$. OLIF, which was first reported by Silvestre et al. ${ }^{[19]}$ in 2012, uses the natural space between the psoas major muscle and the abdominal aorta for the surgical approach and has been widely used in recent years for indirect decompression and interbody fusion in patients with lumbar degenerate disorders due to its advantages of yielding less trauma, less perioperative bleeding and a lower incidence of neurological complications ${ }^{[20-22]}$. Although good clinical results of SA OLIF have been reported ${ }^{[23-25]}$, lumbar degenerate disorders have been reported mostly in elderly patients, and complications such as subsidence and migration of the cage after surgery should not be neglected. For patients with dual-energy X-ray absorptiometry (DEXA) T scores of less than -1.0 , posterior instrumentation is often required ${ }^{[26]}$.

In recent years, scholars have paid increasing attention to the influence of cage position on postoperative cage subsidence. Studies ${ }^{[27-28]}$ have reported that the rigidity of the central region of the endplate is significantly less than that of the peripheral region, which is called the epiphyseal ring, from L1 to S1. Therefore, cage position is an important factor of cage subsidence and endplate collapse. However, the best cage position remains unclear. Barsa et al. ${ }^{[29]}$ found a lower cage subsidence rate near the anterior part of the intervertebral space. Abbushi et al. ${ }^{\left[{ }^{[0]}\right.}$ concluded that the medio-medial cage position shows the highest migration rate, and the lowest migration and highest fusion rate are seen when the cage is in the posteromedial position. Thus, the authors suggested posterior medial cage placement for PLIF. However, Ko et al. ${ }^{\left[{ }^{[1]}\right.}$ found that the rate of cage subsidence increases with a more posterior cage position. A previous study ${ }^{[27]}$ shows that the inferior endplate is approximately $40 \%$ stronger than is the superior endplate, and the subsidence probability of the superior endplate is significantly higher than that of the inferior endplate ${ }^{[32-33]}$. Therefore, we analyzed the stress of the L5 SEP and cage to evaluate the effect of cage position on the biomechanics of the osteoporotic lumbar spine.

In the present study, the ROMs of the SA models and BPSF models were more than $28.29 \%$ and $80.56 \%$ smaller in all motions, respectively. This indicated that both SA and BPSF could provide stability for the surgical segment. The stresses of the L5 SEP and cage were the lowest when the cage was placed in the middle third of the L5 SEP and were the highest when the cage was placed in the posterior third in both the SA models and the BPSF models. This result may be related to the contact area between the cage and the endplate changing according to the cage position during lumbar movement; the larger the contact area was, the smaller the stress. The stress of the fixation was also the lowest in most motions when the cage was placed in the middle third of the L5 SEP, which may be related to the fact that different cage positions affect mechanical properties such as the moment arm and torque of the bilateral pedicle screw fixation. Kim et al. ${ }^{[34]}$ thought that cage positioning and the application of greater compressive load are two reasons for the high risk of cage subsidence. Based on these results, placing the cage in the middle third of the L5 SEP could help reduce the maximum stresses of the L5 SEP, the cage and the fixation, which may be beneficial for reducing the risk of postoperative cage subsidence, endplate collapse and 
fixation fracture, regardless of whether SA or BPSF is performed. This position is consistent with the cage placement recommended in the Guidelines for the clinical application of lumbar oblique lateral interbody fusion $^{[35]}$. The guidelines also point out that whether or not to apply SA technology should be decided on the basis of whether the application of a cage alone can provide sufficient stability ${ }^{[35]}$. In our study, the L4-L5 ROM, the stress of the cage and the stress of the L5 SEP were smaller under all motions in the BPSF models than those of the SA models, especially in flexion and extension. This suggested that the SA method could not provide sufficient stability under some motions. In contrast, BPSF could significantly increase the stability of the surgical segment and reduce the stresses of the cage and L5 SEP in all motions, which could improve the safety of OLIF surgery in osteoporotic lumbar spine. This may be the reason why the BPSF group had a lower incidence of cage settlement than did the SA group in the study by Zeng et al. ${ }^{[25]}$. It is also worth noting that almost all surgical models showed the largest stress values for the cage and endplate in flexion, so few bending movements should be performed after OLIF surgery.

In addition to considering the factors of subsidence when placing the cage, it is also necessary for clinicians to restore sagittal balance and induce sufficient indirect decompression. Kepler et al. ${ }^{[36]}$ concluded that anteroposterior cage placement is an important intraoperative determinant of postoperative alignment; anterior placement leads to larger angles of lordosis, while middle/posterior placement has a minimal effect on sagittal alignment. The authors also thought the rate of new neurologic deficits postoperatively after placement of the cage in the posterior part of the disc space may become significant. Park et al. ${ }^{[37]}$ suggested that placing the cage within the anterior third of the disk space is better for restoring the segmental angle without compromising the extent of indirect neural decompression achieved with LLIF if the height of the cage is large enough. Jin et al[38] found that cages are located mostly in the middle third of the vertebral body, significantly increasing the posterior disk space height and foraminal height compared with those in the anterior cage position. However, another study ${ }^{[39]}$ proposes that increases in disk height and foraminal and canal areas are not dependent on cage positioning within the disk space; as intraoperative placement of a cage in the central portion of the disk is an easier and safer technique, central placement may be preferable in a clinical setting.

According to the clinical experience of our team, SA OLIF is preferred during the first-stage operation, and the degree of improvement in the patients' symptoms and imaging findings observed postoperatively are used to determine whether to perform the two-stage operation combined with posterior pedicle screw fixation. In this study, we recommend that if the images taken between the two operations shows that the cage has migrated toward the posterior part of the disc space, posterior pedicle screw fixation should be performed to reduce the risk of postoperative complications.

In this study, an osteoporotic lumbar spine model was created, and the results were informative. However, our study has some limitations. The FE models were simplified. The residual discs of the surgical segments and musculoskeletal segments were not modeled, and ligaments were replaced with springs, which differ from biological ligaments in terms structure. Furthermore, the lumbar spine varies across 
individuals, and the present study was based on a model of the lumbar spine for analysis, so the results have yet to be validated in a clinical study.

\section{Conclusion}

- The cage position should be selected according to the specific conditions. For the osteoporotic lumbar spine, the cage should be placed in the middle third of the disc space. For the lumbar spine with significant sagittal imbalance, the cage should be placed in the anterior third of the disc space so that it helps to restore sagittal balance and does not affect the extent of indirect decompression. If the cage migrates toward the posterior part of the disc space after the first-stage operation, a second-stage operation combined with posterior pedicle screw fixation should be performed in a timely manner. Compared to SA OLIF, BPSF can provide sufficient stability for the surgical segment in the osteoporotic lumbar spine and may reduce the incidence of postoperative endplate collapse, cage subsidence and posterior internal fixation fracture.

\section{Abbreviations}

OLIF: Oblique lumbar interbody fusion

SA: Stand-alone

BPSF: Bilateral pedicle screw fixation

OP: Osteoporosis

SEP: Superior endplate

ROM: Range of motion

LIF: Lumbar interbody fusion

FE: Finite element

DEXA: dual-energy X-ray absorptiometry

\section{Declarations}

\section{Ethics approval and consent to participate}

This study has been reviewed by Ethic Committee of the Second Hospital of Shanxi Medical University and performed in accordance with the ethical standards laid down in an appropriate version of the 1964 Declaration of Helsinki. The informed consent was obtained from the participant. 
Not applicable.

\section{Availability of data and materials}

The datasets used and/or analysed during the current study are available from the corresponding author on reasonable request.

\section{Competing interests}

The authors declare that they have no competing interests.

\section{Funding}

This study was supported by Natural Science Foundation of Shanxi Province 『201801D121220区.

\section{Authors' contributions}

YCQ and YFW carried out the finite element analysis and drafted the manuscript. BZ, JY and CJX participated in the study design and discussion of the results. YCQ, JQS, JQH and JL constructed the finite element models. All authors read and approved the final manuscript.

\section{Acknowledgements}

Not applicable.

\section{References}

1. Zhang J, Fan S, Fang X, et al. Clinical value of one-level oblique lateral interbody fusion in the treatment of degenerative lumbar disc diseases. Chin J Orthop. 2017;37(2):80-88. doi:10.3760/cma.j.issn.0253-2352.2017.02.003.

2. Ohtori S, Orita S, Yamauchi K, et al. Mini-Open Anterior Retroperitoneal Lumbar Interbody Fusion:Oblique Lateral Interbody Fusion for Lumbar Spinal Degeneration Disease. Yonsei Med J. 2015;56(4):1051-1059. doi:10.3349/ymj.2015.56.4.1051.

3. Ahmadian A, Bach K, Bolinger B, et al. Stand-alone minimally invasive lateral lumbar interbody fusion: multicenter clinical outcomes. J Clin Neurosci. 2015;22(4):740-746. doi:10.1016/j.jocn.2014.08.036.

4. Wang Y, Battié MC, Videman T. A morphological study of lumbar vertebral endplates: radiographic, visual and digital measurements. Eur Spine J. 2012;21(11):2316-2323. doi:10.1007/s00586-0122415-8.

5. Polikeit A, Nolte LP, Ferguson SJ. The effect of cement augmentation on the load transfer in an osteoporotic functional spinal unit: finite-element analysis. Spine (Phila Pa 1976). 2003;28(10):991996. doi:10.1097/01.BRS.0000061987.71624.17. 
6. Zhao X, Du L, Xie Y, Zhao J. Effect of Lumbar Lordosis on the Adjacent Segment in Transforaminal Lumbar Interbody Fusion: A Finite Element Analysis. World Neurosurg. 2018;114:e114-e120. doi:10.1016/j.wneu.2018.02.073.

7. Fang G, Lin Y, Wu J, et al. Biomechanical Comparison of Stand-Alone and Bilateral Pedicle Screw Fixation for Oblique Lumbar Interbody Fusion Surgery-A Finite Element Analysis. World Neurosurg. 2020;141:e204-e212. doi:10.1016/j.wneu.2020.05.245

8. Kim HJ, Kang KT, Chang BS, Lee CK, Kim JW, Yeom JS. Biomechanical analysis of fusion segment rigidity upon stress at both the fusion and adjacent segments: a comparison between unilateral and bilateral pedicle screw fixation. Yonsei Med J. 2014;55(5):1386-1394. doi:10.3349/ymj.2014.55.5.1386.

9. Ruberté LM, Natarajan RN, Andersson GB. Influence of single-level lumbar degenerative disc disease on the behavior of the adjacent segments--a finite element model study. J Biomech. 2009;42(3):341348.doi:10.1016/j.jbiomech.2008.11.024.

10. Chen YL, Lai OJ, Wang Y, Ma WH, Chen QX. The biomechanical study of a modified lumbar interbody fusion-crenel lateral interbody fusion (CLIF): a three-dimensional finite-element analysis. Comput Methods Biomech Biomed Engin. 2020;23(9):548-555. doi:10.1080/10255842.2020.1745784.

11. Moro T, Kikuchi S, Konno S, Yaginuma H. An anatomic study of the lumbar plexus with respect to retroperitoneal endoscopic surgery. Spine (Phila Pa 1976). 2003;28(5):423-428.

doi:10.1097/01.BRS.0000049226.87064.3B.

12. Wang B, Hua W, Ke W, et al. Biomechanical Evaluation of Transforaminal Lumbar Interbody Fusion and Oblique Lumbar Interbody Fusion on the Adjacent Segment: A Finite Element Analysis. World Neurosurg. 2019;126:e819-e824. doi:10.1016/j.wneu.2019.02.164.

13. Xiao Z, Wang L, Gong H, Zhu D. Biomechanical evaluation of three surgical scenarios of posterior lumbar interbody fusion by finite element analysis. Biomed Eng Online. 2012;11:31. Published 2012 Jun 18. doi:10.1186/1475-925X-11-31.

14. Shim CS, Park SW, Lee SH, Lim TJ, Chun K, Kim DH. Biomechanical evaluation of an interspinous stabilizing device, Locker. Spine (Phila Pa 1976). 2008;33(22):E820-E827. doi:10.1097/BRS.0b013e3181894fb1.

15. Zhong ZC, Hung $\mathrm{C}$, Lin HM, Wang $\mathrm{YH}$, Huang $\mathrm{CH}$, Chen $\mathrm{CS}$. The influence of different magnitudes and methods of applying preload on fusion and disc replacement constructs in the lumbar spine: a finite element analysis. Comput Methods Biomech Biomed Engin. 2013;16(9):943-953. doi:10.1080/10255842.2011.645226.

16. Assaker R. Minimal access spinal technologies: state-of-the-art, indications, and techniques. Joint Bone Spine. 2004;71(6):459-469. doi:10.1016/j.jbspin.2004.08.006.

17. Mobbs RJ, Phan K, Malham G, Seex K, Rao PJ. Lumbar interbody fusion: techniques, indications and comparison of interbody fusion options including PLIF, TLIF, MI-TLIF, OLIF/ATP, LLIF and ALIF. J Spine Surg. 2015;1(1):2-18. doi:10.3978/j.issn.2414-469X.2015.10.05. 
18. Yoshihara $\mathrm{H}$, Yoneoka D. National trends in the surgical treatment for lumbar degenerative disc disease: United States, 2000 to 2009. Spine J. 2015;15(2):265-271. doi:10.1016/j.spinee.2014.09.026.

19. Silvestre C, Mac-Thiong JM, Hilmi R, Roussouly P. Complications and Morbidities of Mini-open Anterior Retroperitoneal Lumbar Interbody Fusion: Oblique Lumbar Interbody Fusion in 179 Patients. Asian Spine J. 2012;6(2):89-97. doi:10.4184/asj.2012.6.2.89.

20. Li JX, Phan K, Mobbs R. Oblique Lumbar Interbody Fusion: Technical Aspects, Operative Outcomes, and Complications. World Neurosurg. 2017;98:113-123. doi:10.1016/j.wneu.2016.10.074.

21. Abe K, Orita S, Mannoji C, et al. Perioperative Complications in 155 Patients Who Underwent Oblique Lateral Interbody Fusion Surgery: Perspectives and Indications From a Retrospective, Multicenter Survey. Spine (Phila Pa 1976). 2017;42(1):55-62. doi:10.1097/BRS.0000000000001650.

22. Woods KR, Billys JB, Hynes RA. Technical description of oblique lateral interbody fusion at L1-L5 (OLIF25) and at L5-S1 (OLIF51) and evaluation of complication and fusion rates. Spine J. 2017;17(4):545-553. doi:10.1016/j.spinee.2016.10.026.

23. Mehren C, Mayer HM, Zandanell C, Siepe CJ, Korge A. The Oblique Anterolateral Approach to the Lumbar Spine Provides Access to the Lumbar Spine With Few Early Complications. Clin Orthop Relat Res. 2016;474(9):2020-2027. doi:10.1007/s11999-016-4883-3.

24. Zhu G, Hao Y, Yu L, Cai Y, Yang X. Comparing stand-alone oblique lumbar interbody fusion with posterior lumbar interbody fusion for revision of rostral adjacent segment disease: A STROBEcompliant study. Medicine (Baltimore). 2018;97(40):e12680. doi:10.1097/MD.0000000000012680.

25. Zeng ZY, Xu ZW, He DW, et al. Complications and Prevention Strategies of Oblique Lateral Interbody Fusion Technique. Orthop Surg. 2018;10(2):98-106. doi:10.1111/os.12380.

26. Tempel ZJ, Gandhoke GS, Okonkwo DO, Kanter AS. Impaired bone mineral density as a predictor of graft subsidence following minimally invasive transpsoas lateral lumbar interbody fusion. Eur Spine J. 2015;24 Suppl 3:414-419. doi:10.1007/s00586-015-3844-y.

27. Grant JP, Oxland TR, Dvorak MF. Mapping the structural properties of the lumbosacral vertebral endplates. Spine (Phila Pa 1976). 2001;26(8):889-896. doi:10.1097/00007632-200104150-00012.

28. Lowe TG, Hashim S, Wilson LA, et al. A biomechanical study of regional endplate strength and cage morphology as it relates to structural interbody support. Spine (Phila Pa 1976). 2004;29(21):23892394. doi:10.1097/01.brs.0000143623.18098.e5.

29. Barsa $P$, Suchomel $P$. Factors affecting sagittal malalignment due to cage subsidence in standalone cage assisted anterior cervical fusion. Eur Spine J. 2007;16(9):1395-1400. doi:10.1007/s00586-0060284-8.

30. Abbushi A, Cabraja M, Thomale UW, Woiciechowsky C, Kroppenstedt SN. The influence of cage positioning and cage type on cage migration and fusion rates in patients with monosegmental posterior lumbar interbody fusion and posterior fixation. Eur Spine J. 2009;18(11):1621-1628. doi:10.1007/s00586-009-1036-3. 
31. Ko MJ, Park SW, Kim YB. Effect of Cage in Radiological Differences between Direct and Oblique Lateral Interbody Fusion Techniques. J Korean Neurosurg Soc. 2019;62(4):432-441. doi:10.3340/jkns.2018.0142.

32. Tohmeh AG, Khorsand D, Watson B, Zielinski X. Radiographical and clinical evaluation of extreme lateral interbody fusion: effects of cage size and instrumentation type with a minimum of 1-year follow-up. Spine (Phila Pa 1976). 2014;39(26):E1582-E1591. doi:10.1097/BRS.0000000000000645.

33. Le TV, Baaj AA, Dakwar E, et al. Subsidence of polyetheretherketone intervertebral cages in minimally invasive lateral retroperitoneal transpsoas lumbar interbody fusion. Spine (Phila Pa 1976). 2012;37(14):1268-1273. doi:10.1097/BRS.0b013e3182458b2f.

34. Kim MC, Chung HT, Cho JL, Kim DJ, Chung NS. Subsidence of polyetheretherketone cage after minimally invasive transforaminal lumbar interbody fusion. J Spinal Disord Tech. 2013;26(2):87-92. doi:10.1097/BSD.0b013e318237b9b1.

35. Chinese Medical Association Society of Orthopaedic Spine Group. Guidelines for the clinical application of lumbar oblique lateral interbody fusion. Chin J Orthop. 2020;40(8):459-468. doi:10.3760/cma.j.cn121113-20200309-00142.

36. Kepler CK, Huang RC, Sharma AK, et al. Factors influencing segmental lumbar lordosis after lateral transpsoas interbody fusion. Orthop Surg. 2012;4(2):71-75. doi:10.1111/j.1757-7861.2012.00175.x.

37. Park SJ, Lee CS, Chung SS, Kang SS, Park HJ, Kim SH. The Ideal Cage Position for Achieving Both Indirect Neural Decompression and Segmental Angle Restoration in Lateral Lumbar Interbody Fusion (LLIF). Clin Spine Surg. 2017;30(6):E784-E790. doi:10.1097/BSD.0000000000000406.

38. Jin J, Ryu KS, Hur JW, Seong JH, Kim JS, Cho HJ. Comparative Study of the Difference of Perioperative Complication and Radiologic Results: MIS-DLIF (Minimally Invasive Direct Lateral Lumbar Interbody Fusion) Versus MIS-OLIF (Minimally Invasive Oblique Lateral Lumbar Interbody Fusion). Clin Spine Surg. 2018;31(1):31-36. doi:10.1097/BSD.0000000000000474.

39. Marulanda GA, Nayak A, Murtagh R, Santoni BG, Billys JB, Castellvi AE. A cadaveric radiographic analysis on the effect of extreme lateral interbody fusion cage placement with supplementary internal fixation on indirect spine decompression. J Spinal Disord Tech. 2014;27(5):263-270. doi:10.1097/BSD.0b013e31828f9da1.

\section{Figures}



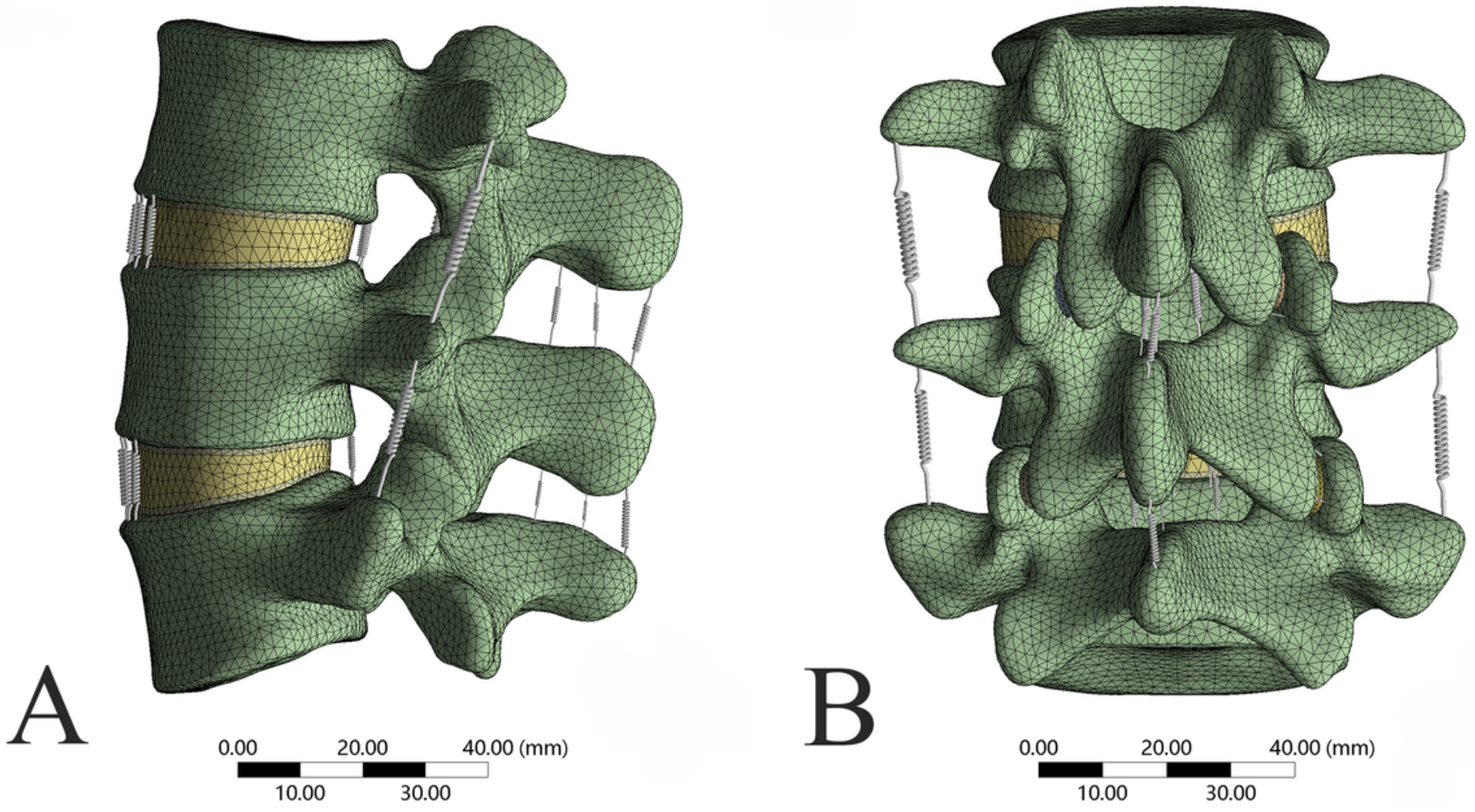

Figure 1

Lateral (A)and posterior (B) views of the L3-L5 FE model.

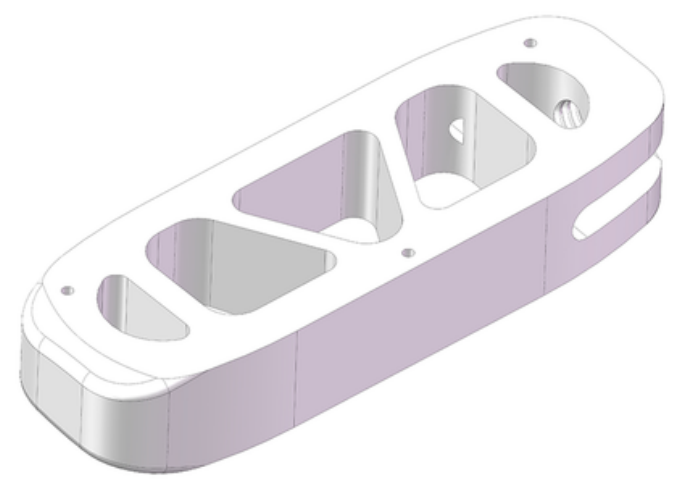

A
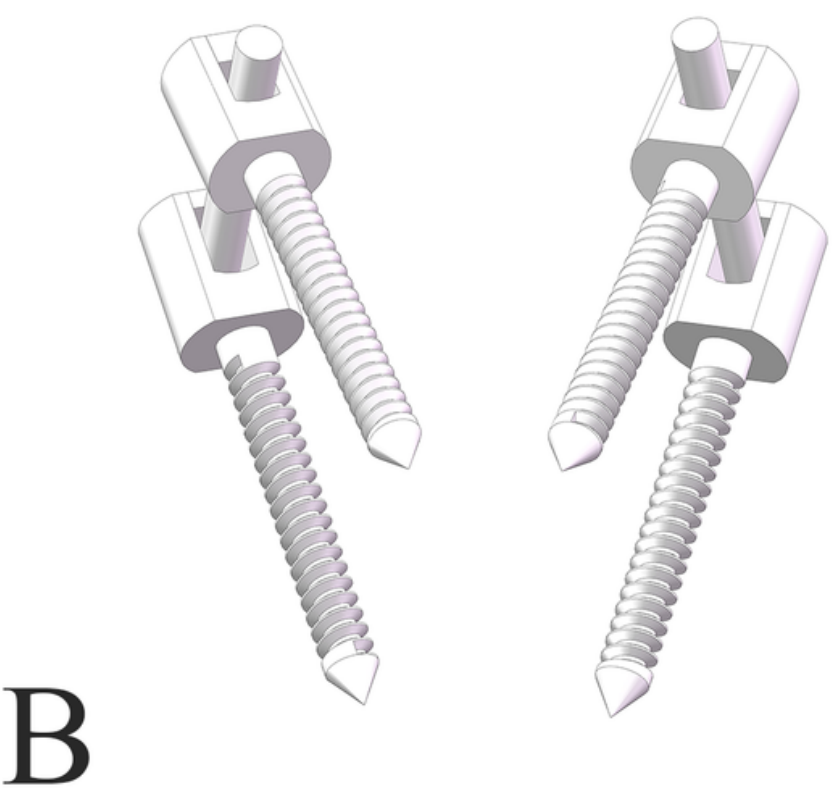

Figure 2

The models of the cage (A) and bilateral pedicle screw fixation (B). 


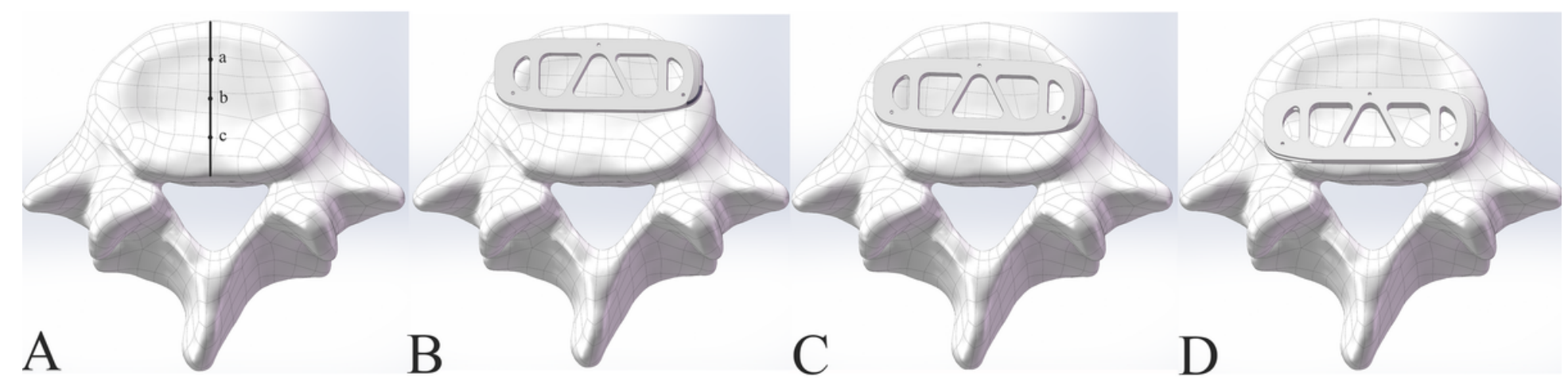

Figure 3

Schematic diagram of the cage position. (A)The L5 SEP was equally divided into four parts, and the demarcation points were recorded as $a, b$ and $c$. The cage was placed in the anterior third (B), middle third (C) and posterior third (D) of the L5 SEP.

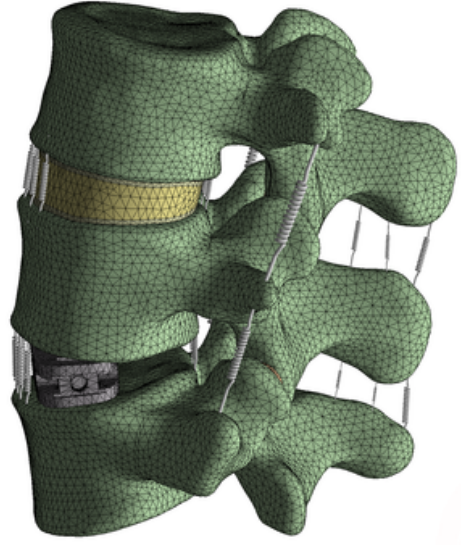

A

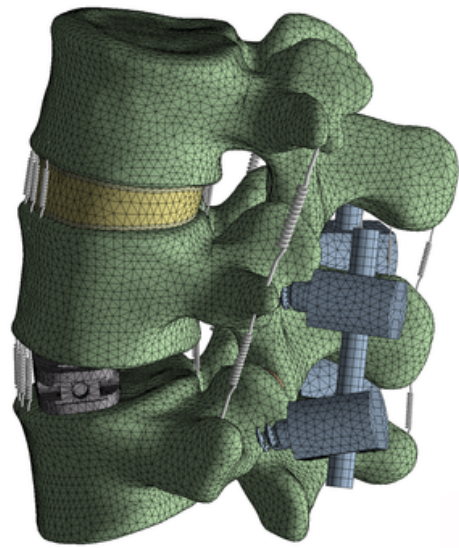

$\mathrm{D}$

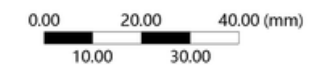

$\stackrel{0.00}{10.00} \frac{20.00}{30.00} 40.00(\mathrm{~mm})$

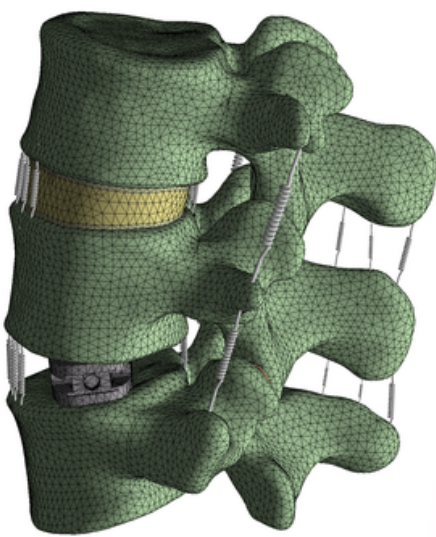

$\mathrm{B}$
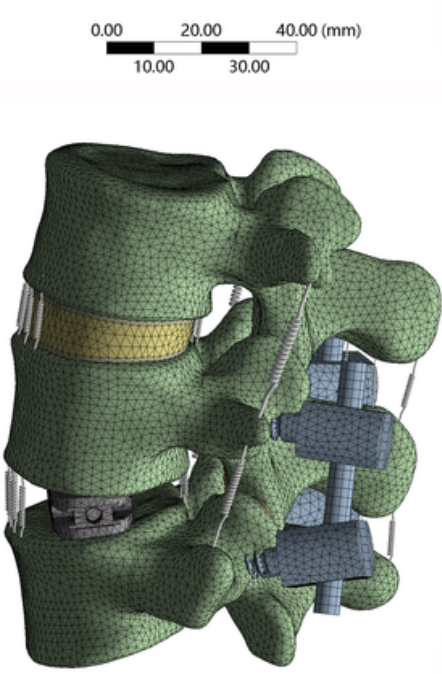

$\mathrm{E}$

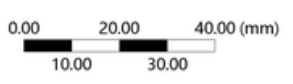

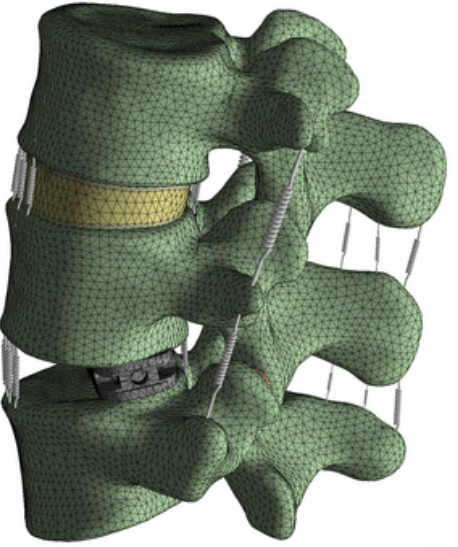

$\mathrm{C}$
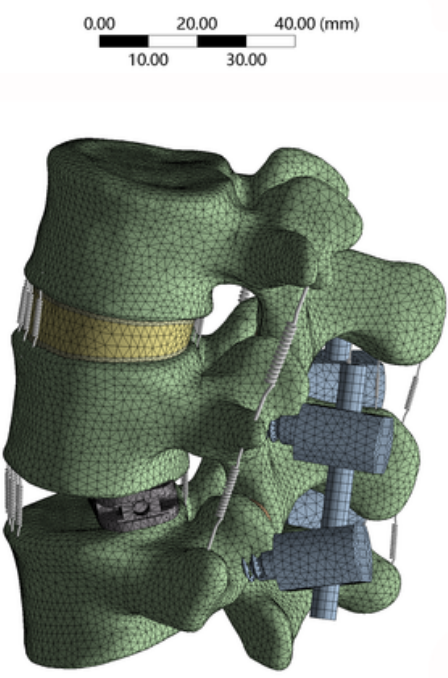

$\mathrm{F}$ $\underbrace{200.00}_{10.00} \underbrace{40.00(\mathrm{~mm})}_{30.00}$

Figure 4 
Six OLIF FE models of different cage positions with SA and BPSF. (A)SA1 (anterior third of the L5 SEP; SA) ; (B) SA2 (middle third of the L5 SEP; SA) ; (C) SA3 (posterior third of the L5 SEP; SA) ; (D) BPSF1 (anterior third of the L5 SEP; BPSF) ; (E) BPSF2 (middle third of the L5 SEP; BPSF) ; (F) BPSF3 (posterior third of the L5 SEP; BPSF).

$\square$ Present study $\square$ Shim et al. $\square$ Zhong et al.

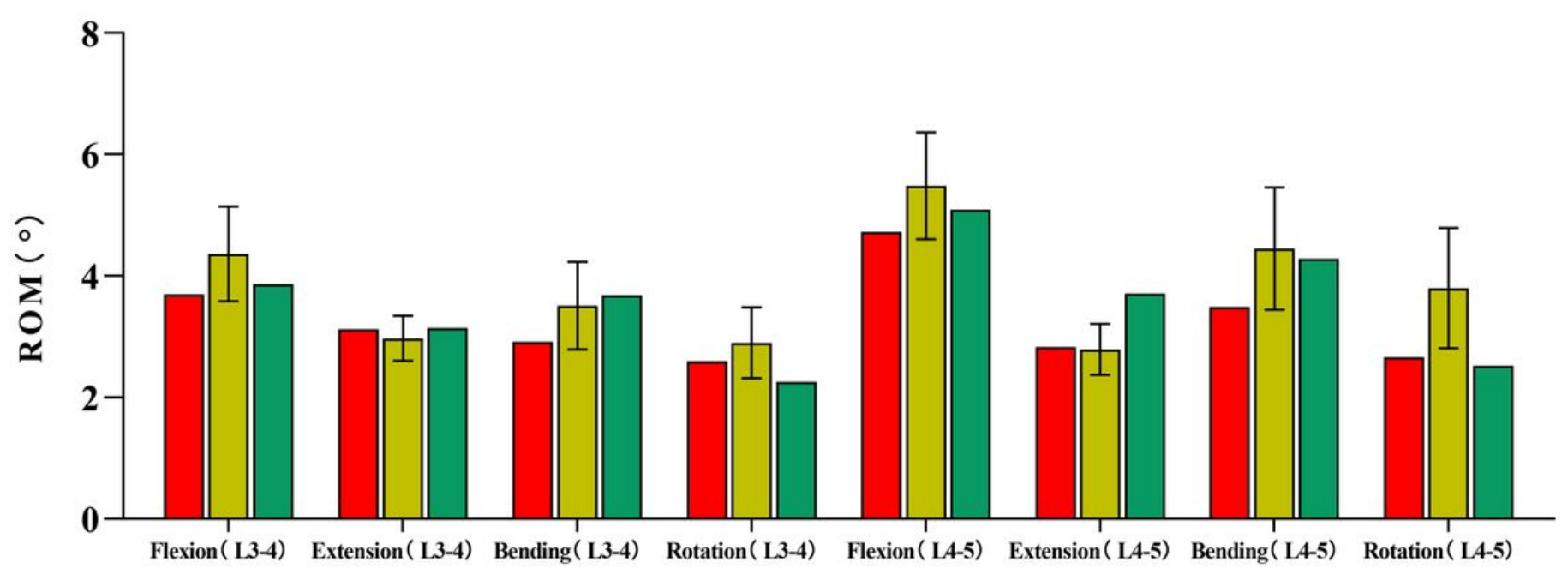

Figure 5

Comparison of ROM between the normal intact FE model and the results of previous studies.

$\square \mathrm{OP} \square \mathrm{SA} 1 \square \mathrm{SA} 2 \square \mathrm{SA} 3 \square$ BPSF1 $\square$ BPSF2 $\square$ BPSF3

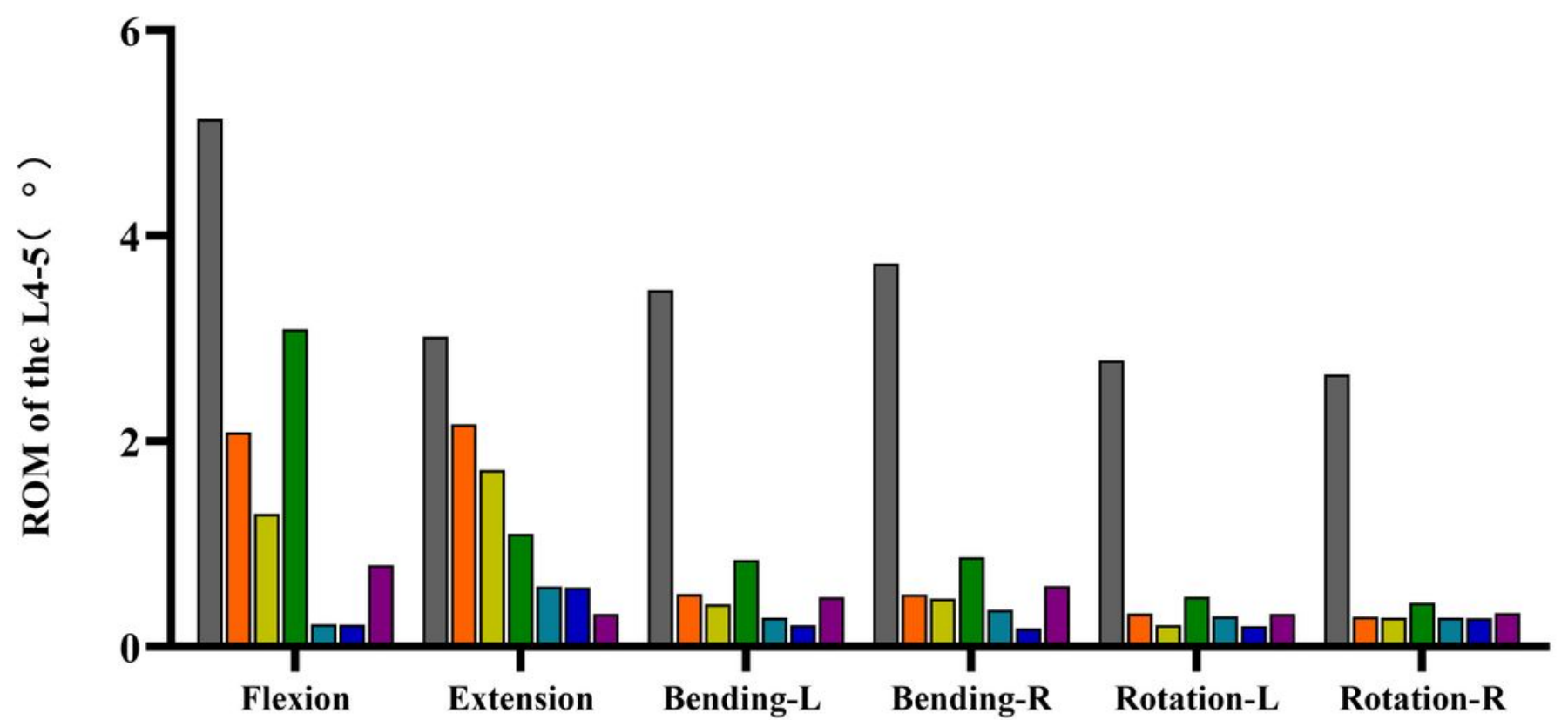

Figure 6 


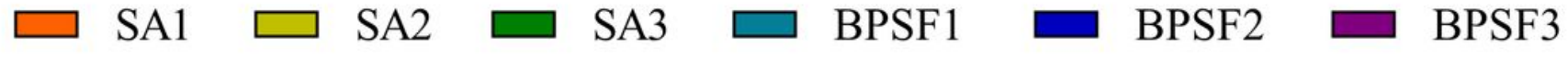

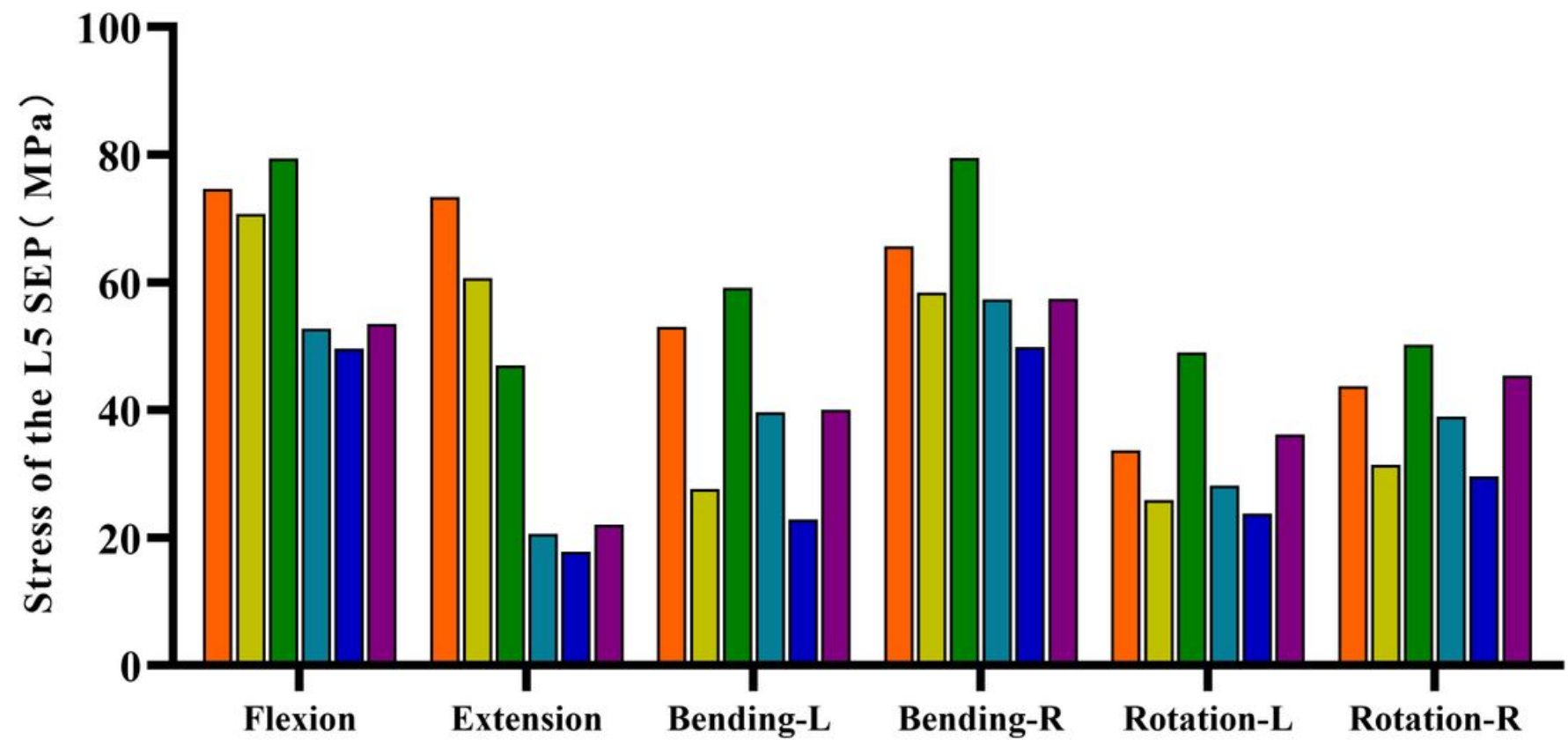

Figure 7

The maximum von Mises stress of the L5 SEP in the OLIF models.

B: Flexion

Stress of the LS SEP

Type: Equivalent (von-Mises) Stress

Unit: $\mathrm{MPa}$

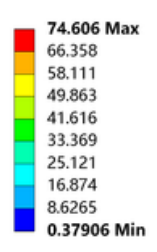

A

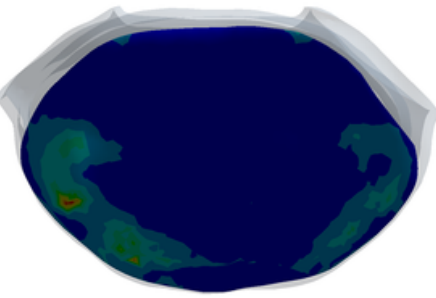

B: Flexion

Stress of the L5 SEP

Type: Equivalent (von-Mises) Stress

Unit: $\mathrm{MPa}$
Time: 1

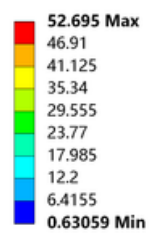

D



B: Flexion

Stress of the LS SEP

Type: Equivalent (von-Mises) Stress

Unit: MPa
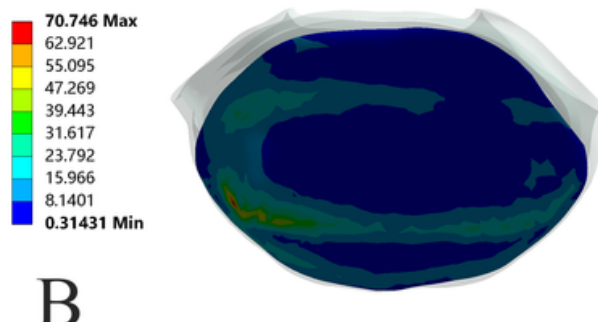

B: Flexion

Type: Equivalent (von-Mises) Stress

Unit: MPa
Time: 1

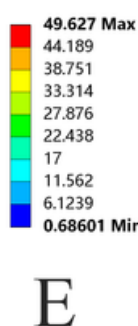

E

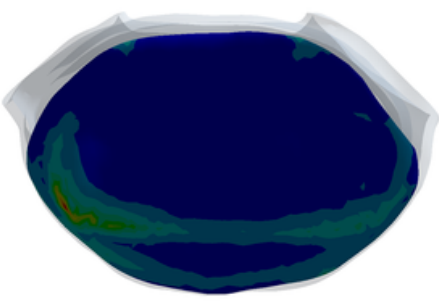

B: Flexion

Type: Equivalent (von-Mises) Stress

Unit. MPa
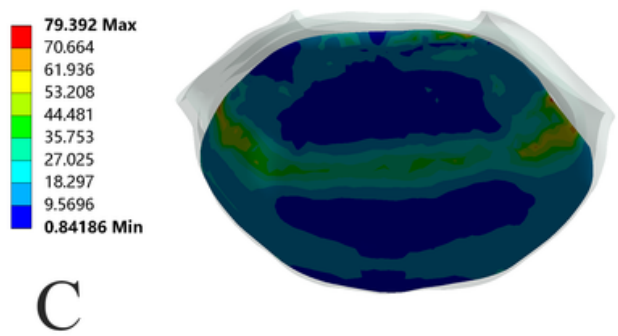

B: Flexion

Type: Equivalent (von-Mises) Stress

Unit: MPa
Time: 1
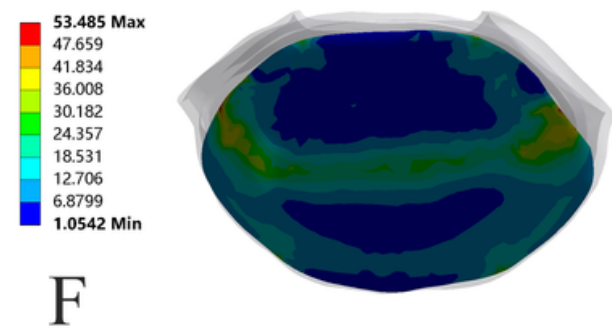

Figure 8 
Cloud map of L5 SEP stress in the OLIF models in flexion: (A) SA1, (B) SA2, (C) SA3, (D) BPSF1, (E) BPSF2 and (F) BPSF3.

$\square$ SA1 $\square$ SA2 $\square$ SA3 $\square$ BPSF1 $\square$ BPSF2 $\square$ BPSF3

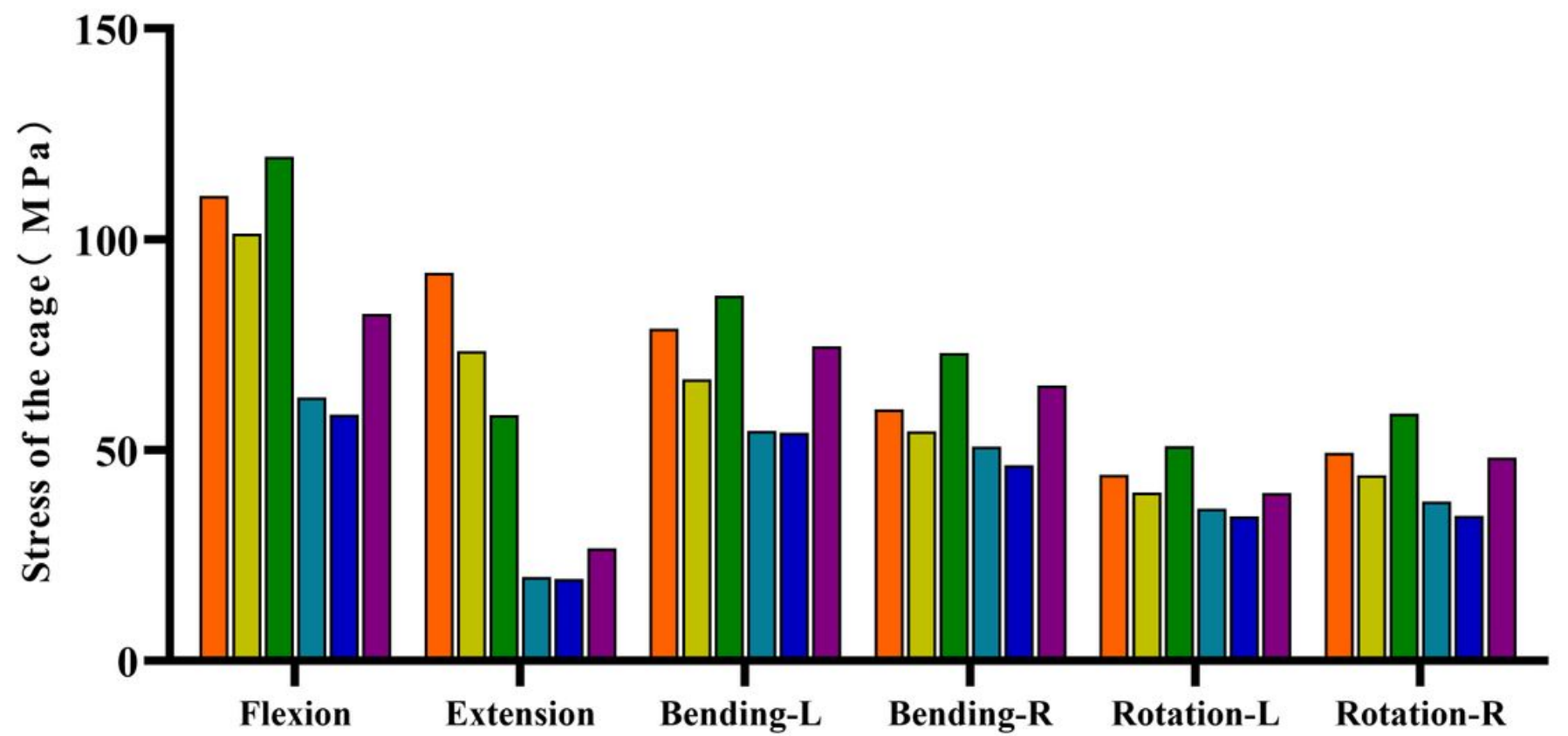

Figure 9

The maximum von Mises stress of the cage in the OLIF models.

B: Flexion

Stress of the cage

Type: Equivalent (von-Mises) Stress

Unit: MPa

110.3 Max

$110.3 \mathrm{M}$
98.066

85.828

73.59
61.351

61.351
49.113

49.11

36.875

24.636

12.398

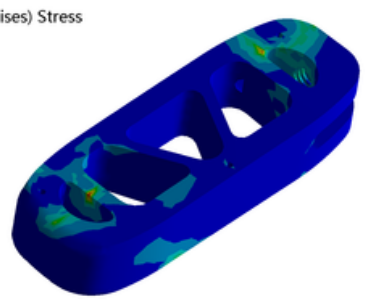

A

B: Flexion

Stress of the cage

Type: Equivalent (von-Mises) Stress

Unit: MPa
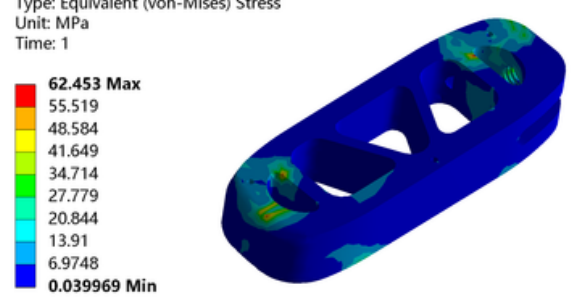

D
B: Flexion

Type: Equivalent (von-Mises) Stress

Unit: MPa

Time: 1

101.36 Max

90.113

78.865
67.616

67.616
56.368
-45.12

56.368
45.12
33.871

45.12
33.871
22.623

22.623
-11.375

11.375

B

B: Flexion

Stress of the cage

Type: Equivalent (von-Mises) Stress

Unit: $\mathrm{MPa}$

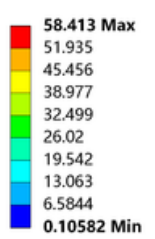

E

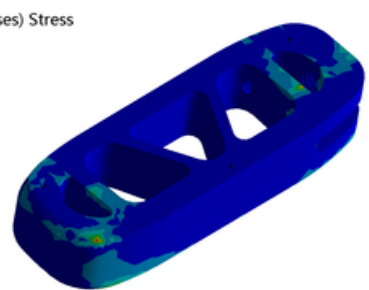

B: Flexion

Stress of the cage

Type: Equivalent (von-Mises) Stress

Unit: MPa

119.53 Max

$\mathbf{1 1 9 . 5 3}$ Max
106.26

93
79.735

79.735
66.47

66.47
53.206

53.206
39.941
25.676

26.676

13.412
0.14681

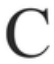

ype. Equivalent (von-Mise

Unit: MP
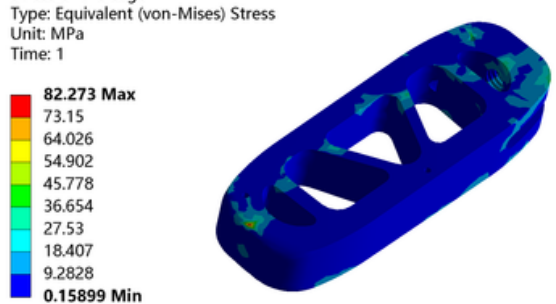

F 
Cloud map of cage stress in the OLIF models in flexion: (A) SA1, (B) SA2, (C) SA3, (D) BPSF1, (E) BPSF2 and (F) BPSF3.

\section{$\begin{array}{lll}\text { BPSF1 BPSF2 } & \text { BPSF3 }\end{array}$}



Figure 11

The maximum von Mises stress of fixation in the BPSF models.

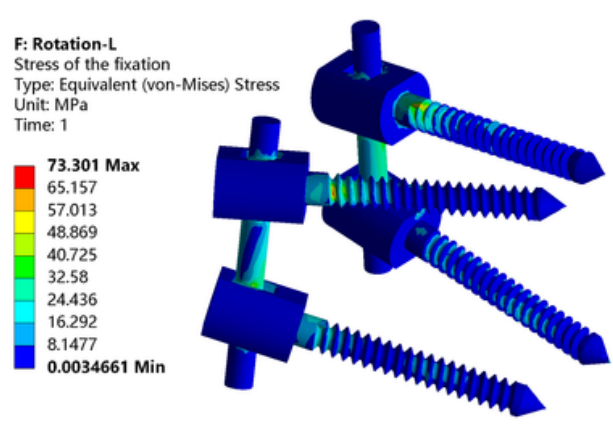

A

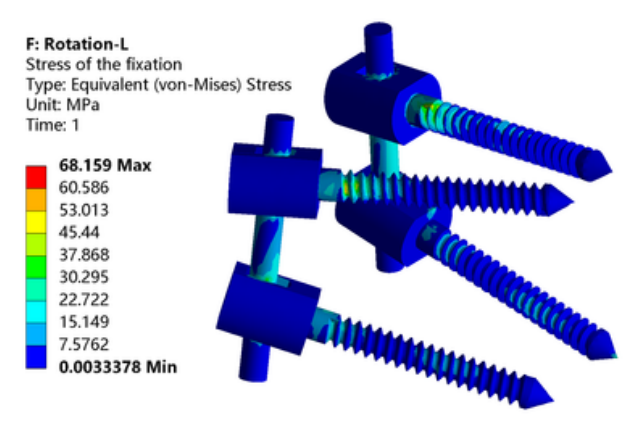

B

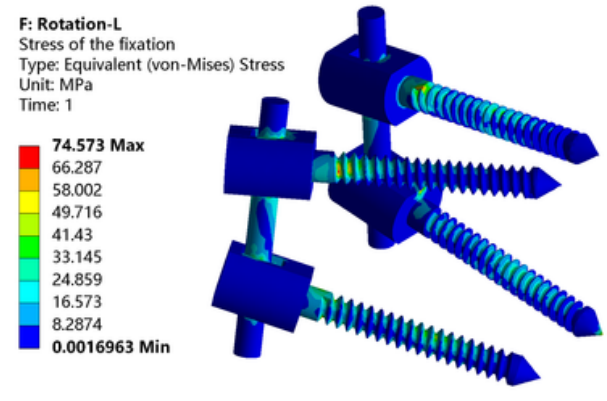

C

Figure 12

Cloud map of fixation stress in the BPSF models in left axial rotation: (A) BPSF1, (B) BPSF2 and (C) BPSF3. 